\title{
Night-time blood pressure: a role in the prediction and prevention of diabetes?
}

\author{
Martin K. Rutter ${ }^{1,2,3}$ \\ Received: 12 October 2015 / Accepted: 6 November 2015 / Published online: 2 December 2015 \\ (C) Springer-Verlag Berlin Heidelberg 2015
}

\begin{abstract}
This commentary discusses and reviews the implications of two studies published in this issue of Diabetologia by Hermida et al (DOIs: 10.1007/s00125-015-3748-8 and $10.1007 / \mathrm{s} 00125-015-3749-7)$ suggesting that high nocturnal blood pressure could have a role in the prediction of diabetes and act as a therapeutic target to prevent diabetes. This overview addresses the clinical implications of this work and new research that is likely to advance this field. It also provides a framework for interpreting claims of causality from observational studies and clinical trials.
\end{abstract}

Keywords Antihypertensive therapy - Chronobiology · Hypertension · Prediction · Prevention · Type 2 diabetes

Many biological processes display periodic variation over a $24 \mathrm{~h}$ cycle, including hormone levels, inflammatory mediators and autonomic function. There is increasing awareness that disordered circadian rhythms may be highly relevant in the pathophysiology of many diseases, including diabetes [1]. This commentary discusses and reviews the implications of two studies published by the same research group in this issue of Diabetologia. These studies explore the possibility that high nocturnal blood pressure could have a role in the prediction of diabetes and act as a therapeutic target to prevent diabetes [2, 3].

Martin K. Rutter

Martin.Rutter@manchester.ac.uk

1 Endocrinology and Diabetes Research Group, Faculty of Medical and Human Sciences, University of Manchester, Manchester, UK

2 Manchester Diabetes Centre, 193 Hathersage Road, Manchester, UK

3 Manchester Academic Health Science Centre, Central Manchester University Hospitals NHS Foundation Trust, Manchester M13 0JE, UK

\section{Observational study}

In this study [2], the prognostic value of $48 \mathrm{~h}$ ambulatory blood pressure was assessed as a predictor of diabetes in 2, 656 adults without diabetes at baseline. Over 5.9 years, 190 $(7.2 \%)$ of the participants developed diabetes. The study was nested within the randomised trial of nocturnal blood pressure lowering [3], which will be discussed later. Activity monitors provided an objective measure of sleep onset and duration. After adjusting for differences in baseline age, waist circumference, fasting glucose and kidney disease, higher sleep-time blood pressure and failure of blood pressure to fall at night were the strongest predictors of incident diabetes.

This original study represents an important advance because it could lead to changes in clinical practice. However, it has some limitations. First, regarding clinical utility, the authors show statistical associations between nocturnal blood pressure and incident diabetes. However, we are not provided with the optimal blood pressure threshold for prediction or the number needed to screen to predict an additional case of diabetes. It would also be useful to know the added value of measuring nocturnal blood pressure to predict diabetes beyond that achieved using methods such as QDiabetes (http:// www.qdiabetes.org), which uses readily available clinical data. Second, it appears that diabetes was detected at baseline and follow-up by history and fasting plasma glucose values. It is unclear whether having diabetes diagnosed by $\mathrm{HbA}_{1 \mathrm{c}}$ might have influenced the results. Third, using nighttime blood pressure to predict incident diabetes in the setting of a clinical trial that modified night-time blood pressure might also be problematic. However, the authors have been able to reassure us on this because the prognostic role of nighttime blood pressure on diabetes risk was not influenced by use of antihypertensive therapy or by the time of day when any antihypertensive therapy was taken. 
Finally, in their observational study, the authors suggest that nocturnal blood pressure could be causally linked to diabetes. Observational studies exploring causality often struggle with issues of reverse causality and confounding, and this one is no exception. Let us consider reverse causality first. People with diabetes were excluded at baseline based on clinical history and fasting plasma glucose. Therefore, some people with undiagnosed diabetes might have been included. Through mechanisms, perhaps involving autonomic dysfunction, these individuals could have developed raised nocturnal blood pressure that was evident at baseline. It would seem likely that many of those with undiagnosed diabetes at baseline would be subsequently diagnosed during follow-up, giving the impression that raised nocturnal blood pressure preceded the development of diabetes, when in fact the reverse would be true. Non-diabetic levels of hyperglycaemia might also cause nocturnal hypertension through the same pathophysiological processes, suggesting that nocturnal hypertension causes diabetes. But again, the pathophysiology would be working in the opposite direction from the perspective of causation (hyperglycaemia causing nocturnal hypertension).

The second issue relating to causation is confounding. The authors adjusted their analysis for five potential confounding variables but did not include others such as obstructive sleep apnoea and triacylglycerols. Confounding due to unmeasured variables such as insulin resistance, stress, depression, reduced physical activity and even statin medication could also be relevant. The authors reported previously that nocturnal blood pressure-lowering reduced cardiovascular events [4]. Therefore, those with higher nocturnal blood pressures would have had more cardiovascular events and most likely be prescribed more within-study statins, which could have modestly increased diabetes risk.

In summary, the observational study provides original data suggesting that nocturnal hypertension could help stratify patients for diabetes risk, but further work is required before this can be recommended in clinical practice. Regarding the case for causality, there are concerns about reverse causation and confounding, which erode these arguments.

\section{Interventional study}

The 'gold standard' test to evaluate the effectiveness of an intervention is the double-blind placebo-controlled clinical trial. In this issue, Hermida et al [3] present the results of a prospective, randomised, open-label, blinded endpoint trial testing the hypothesis that taking antihypertensive medication at bedtime lowers diabetes risk compared with taking therapy on wakening. Although the study falls short of having the 'gold standard' design, the results are potentially exciting and suggest that bedtime administration of antihypertensive treatment could reduce the risk of developing diabetes.
In summary, 2,012 non-diabetic hypertensive participants were randomised to receive at least one of their antihypertensive medications at bedtime vs taking all their antihypertensive medication in the morning. If blood pressure control was inadequate, additional medications could be added according to the randomised dosing pattern (more in the morning, vs more at night). Over $\sim 6$ years, 171 participants $(8.5 \%)$ developed diabetes. Participants who received at least one antihypertensive at bedtime had lower night-time blood pressure and a substantially lower risk for developing diabetes (event rate of $4.8 \%$ vs. $12.1 \%$ for bedtime vs morning treatment, respectively; adjusted HR 0.43, 95\% CI 0.31, 0.61). The lower risk for developing diabetes was evident in those receiving bedtime regimens including ACE inhibitors, angiotensin receptor blocking agent and long-acting $\beta$-blockers (mostly Nebivolol) but not significant for $\alpha$-blockers, diuretics or calcium channel blockers.

This is an important trial because it tested a clinically important hypothesis but it has several important limitations that influence its interpretation.

First, it appears that incident diabetes was not a specified outcome when the trial was registered (NCT00295542) or first described [5], and therefore the data presented should be considered a post hoc analysis. After excluding 688 individuals with type 2 diabetes at baseline, the size of the randomised subgroups were slightly different (1,029 vs 983 participants in the morning and night-time medication groups, respectively) but baseline characteristics were not significantly different. The authors appropriately adjusted the risk for incident diabetes for the minor non-significant between-group differences in factors that could have influenced diabetes risk. However, we should be clear that this trial was originally set up to assess incident cardiovascular events and not diabetes.

Second, the trial had an open-label design, which means that patients were aware of their treatment allocation (night vs morning dosing). We know that lifestyle interventions can have powerful effects on diabetes risk. It is easy to imagine that, during the trial, individuals, with knowledge of their differing treatment allocation, could adopt different lifestyle patterns. For example, we are provided with no information about withingroup changes in weight, diet, physical activity, sleep and night-time eating during the study. A randomised, double-blind, placebo-controlled clinical trial design would have had important advantages over the trial presented.

Finally, the diagnosis of diabetes relied on serial morning fasting plasma glucose tests. It is possible that the effect of the intervention (night-time medication) might have had a larger effect on morning fasting glucose than on average glucose levels. Serial screening for diabetes using $\mathrm{HbA}_{1 \mathrm{c}}$ would have been ideal, but this was not done, presumably because incident diabetes was a late addition as a trial endpoint. Thus, 
although the trial is of significant interest, some aspects of its design lead to concern about biases that could influence the conclusions.

\section{Clinical and research implications}

It would seem premature for these new data to lead to major changes in clinical care such as the widespread prescribing of all antihypertensive medication night-time. However, the results are provocative and, if confirmed, could have far-reaching implications. These data should at least stimulate further studies including mechanistic studies focusing on pathophysiological mechanisms linking nocturnal blood pressure to incident diabetes and well-designed double-blind randomised controlled trials.

\section{The case for causality}

In 1965 Sir Austin Bradford Hill described nine useful criteria for assessing whether an observed relationship between two variables is causal: strength of association; consistency; specificity; temporality; biological gradient; plausibility; coherence; experiment and analogy [6]. Data presented here go some way to satisfy some of these criteria (strength of association; temporality; biological gradient; and experiment) but as we have seen, the evidence is less than conclusive.

Replication of these studies and further trials will be required to demonstrate consistency. Supporting this we have evidence from the Heart Outcomes Prevention Evaluation (HOPE) trial which showed that night-time Ramipril reduced the risk of incident diabetes by $34 \%$ [7]. Mechanistic studies might provide additional data on plausibility and coherence. There are already plausible mechanisms involving ACE inhibitors, angiotensin receptor blockers and Nebivolol that could influence the risk of diabetes through effects on glucose, insulin resistance, lipids, the renin-angiotensin system and sleep [8-10].

\section{Conclusion}

The authors have presented provocative data that could have clinical implications - particularly for the prediction of diabetes. In the exploration of causality, the observational data are potentially limited by reverse causation and confounding and the fact that the trial data fall somewhat short of providing conclusive proof of the benefit of intervention.

Acknowledgements The author would like to thank David Ray, University of Manchester, for critical discussion of the manuscript. The author acknowledges support from Manchester Academic Health Science Centre.

Funding MKR is supported by the University of Manchester and Central Manchester University Hospitals NHS Foundation Trust.

Duality of interest The author declares that there is no duality of interest associated with this work.

Contribution statement The author was responsible for the conception, design, drafting, revision and approval of the article.

\section{References}

1. Huang W, Ramsey KM, Marcheva B, Bass J (2011) Circadian rhythms, sleep, and metabolism. J Clin Invest 121:2133-2141

2. Hermida RC, Ayala DE, Mojón A, Fernández JR (2015) Sleep-time BP: prognostic marker of type 2 diabetes and therapeutic target for prevention. Diabetologia. doi:10.1007/s00125-015-3748-8

3. Hermida RC, Ayala DE, Mojón A, Fernández JR (2015) Bedtime ingestion of hypertension medications reduces the risk of new-onset type 2 diabetes: a randomised controlled trial. Diabetologia. doi: 10 . 1007/s00125-015-3749-7

4. Hermida RC, Ayala DE, Mojon A, Fernandez JR (2011) Decreasing sleep-time blood pressure determined by ambulatory monitoring reduces cardiovascular risk. J Am Coll Cardiol $58: 1165-1173$

5. Hermida RC (2007) Ambulatory blood pressure monitoring in the prediction of cardiovascular events and effects of chronotherapy: rationale and design of the MAPEC study. Chronobiol Int 24:749-775

6. Hill AB (1965) The environment and disease: association or causation? Proc R Soc Med 58:295-300

7. Bosch J, Lonn E, Pogue J, Arnold JM, Dagenais GR, Yusuf S (2005) Long-term effects of ramipril on cardiovascular events and on diabetes: results of the HOPE study extension. Circulation 112:1339-1346

8. Li L, Luo Z, Yu H et al (2013) Telmisartan improves insulin resistance of skeletal muscle through peroxisome proliferator-activated receptor-delta activation. Diabetes 62:762-774

9. McFarlane SI, Kumar A, Sowers JR (2003) Mechanisms by which angiotensin-converting enzyme inhibitors prevent diabetes and cardiovascular disease. Am J Cardiol 91:30H-37H

10. Van Bortel LM (2010) Efficacy, tolerability and safety of nebivolol in patients with hypertension and diabetes: a post-marketing surveillance study. Eur Rev Med Pharmacol Sci 14:749-758 